\title{
Utilización de chamota posconsumo en la formulación de una pasta refractaria como sustitución de la alúmina
}

\author{
Saúl Idarraga-Giraldo; Sebastián Figueroa-Calle; Fabio Alonso Vargas-Bermúdez²; \\ Carlos Mario Mesa-Toro²; Santiago Gil-Durán ${ }^{3 *}$ \\ ${ }^{1}$ Grupo de Investigación en Materiales de Ingeniería, Escuela de Ingeniería, Universidad EAFIT. Medellín, \\ Colombia. \\ ${ }^{2}$ Departamento de Investigación y desarrollo, Gamma|Erecos ®. Medellín, Colombia. \\ ${ }^{3}$ Grupo de Investigación Calidad, Metrología y Producción, Instituto Tecnológico Metropolitano. Medellín, \\ Colombia. \\ *santiagogil@itm.edu.co
}

\begin{abstract}
Resumen
El desarrollo de los materiales cerámicos refractarios ha sido derivado de las industrias que utilizan altas temperaturas en sus procesos. El uso de material posconsumo para la formulación de pastas refractarias es de interés para el desarrollo sostenible con impacto económico y ambiental. El objetivo de este trabajo es estudiar el efecto de la adición de chamota posconsumo en una pasta refractaria como reemplazo de alúmina. La chamota fue obtenida a partir de ladrillos aislantes refractarios porosos que cumplieron su ciclo de vida en la industria cerámica. Estos fueron sometidos a un proceso de conminución utilizando un molino de bolas hasta llevarlo a pasante malla 50. La chamota se caracterizó mediante Fluorecencia de rayos $X$. Se formularon cuatro mezclas y se sinterizaron a $1600^{\circ} \mathrm{C}$. Se evaluó el cono pirométrico (ASTM C-24), la densidad y la porosidad (ASTM C-20), la contracción lineal y dilatometría. Los resultados indican que la adición de chamota posconsumo disminuye el cono pirométrico del refractario obtenido; la muestra que no contiene chamota tiene un cono superior a 36 y la muestra de mayor contenido tiene un cono de 32, los cuales son adecuados para el uso como ladrillo refractario. Con la adición de chamota se aumentó la densidad de las muestras y se disminuyó la porosidad y la contracción. Finalmente, en el ensayo de dilatometría se evidencian cambios en la curva dilatometría que se atribuyen a una mayor formación de fase líquida y mulita, debido al aporte que hace la sílice y el $\mathrm{K}_{2} \mathrm{O}$ presentes en la chamota.
\end{abstract}

Palabras clave: Chamota Posconsumo; Refractarios; Dilatometría Cono Pirométrico Equivalente. 


\title{
Use of chamotte post-consumption in the formulation of a refractory paste as a replacement of the aluminum oxide
}

\begin{abstract}
The development of refractory ceramic materials has been derived from industries that use high temperatures in their processes. The use of post-consumer material for the formulation of refractory pastes is of interest for sustainable development with economic and environmental impact. The objective of this work is to study the effect of the addition of post-consumption chamotte in a refractory paste as an alumina replacement. The chamotte was obtained from porous refractory insulating bricks that fulfilled their life cycle in the ceramic industry. These were subjected to a comminution process using a ball mill until it was passed through 50 mesh. The chamotte was characterized by X-ray diffraction. Four mixtures were formulated and sintered at $1600^{\circ} \mathrm{C}$. Pyrometric cone (ASTM C-24), density and porosity (ASTM C-20), linear contraction and dilatometry were evaluated. The results indicate that the addition of postconsumption chamotte reduces the pyrometric cone of the refractory obtained, the sample that does not contain chamotte has a cone over 36 and the sample of higher content has a cone 32, which are suitable for use as a refractory brick. With the addition of chamotte, the density of the samples was increased and the porosity and contraction decreased. Finally, in the dilatometry test, changes in the dilatometric curve are evidenced, which are attributed to a greater formation of liquid phase and fines due to the contribution made by silica and $\mathrm{K}_{2} \mathrm{O}$ present in the chamotte.
\end{abstract}

Keywords: Post-Consumption Chamotte; Refractories; Pyrometric Cone Equivalent Dilatometry.

\section{Uso do chamotte pós-consumo na formulação de uma pasta refratária em substituição da alumina}

\begin{abstract}
Resumo
O desenvolvimento de materiais cerâmicos refratários é derivado de indústrias que utilizam altas temperaturas em seus processos. O uso de material pós-consumo para a formulação de pastas refratárias é de interesse para o desenvolvimento sustentável com impacto econômico e ambiental. O objetivo deste trabalho é estudar o efeito da adição de chamotte pós-consumo em uma pasta refratária como substituição de alumina. A chamotte foi obtida a partir de tijolos isolantes refratários porosos que cumpriram seu ciclo de vida na indústria cerâmica. Estes foram submetidos a um processo de trituração usando um moinho de bolas até passar pela malha 50. A chamotte foi caracterizada por difração de raios X. Quatro misturas foram formuladas e sinterizadas a $1600{ }^{\circ} \mathrm{C}$. Foram avaliados cone cromático (ASTM C-24), densidade e porosidade (ASTM C-20), contração linear e dilatometria. Os resultados indicam que a adição de chamotte pós-consumo reduz o cone pirrométrico do refratário obtido, a amostra que não contém chamotte tem um cone maior que 36 e a amostra de maior conteúdo possui um cone de 32, adequado para uso como tijolo refratário Com a adição de chamotte, a densidade das amostras aumentou e a porosidade e contração diminuíram. Finalmente, no teste de dilatometria, são evidenciadas alterações na curva dilatométrica, atribuídas a uma maior formação de fase líquida e finas devido à contribuição da sílica e do $\mathrm{K}_{2} \mathrm{O}$ presente na chamotte.
\end{abstract}

Palavras-chave: Chamotte Pós-Consumo; Refratários; Dilatometria Pirométrica de Cone Equivalente. 


\section{Introducción}

Los refractarios son esenciales para las industrias que trabajan con altas temperaturas; estos pueden soportar extremas condiciones conservando sus propiedades mecánicas [1]. La producción mundial anual de refractarios se estima entre 35 y 40 millones de toneladas por año [2]. Estos volúmenes tan altos de producción generan dos problemas ambientales. El primero está relacionado con el uso exhaustivo de recursos naturales no renovables, y el segundo consiste en la producción de materiales posconsumo que se deben disponer adecuadamente [3]. El reciclaje de refractarios posconsumo no ha recibido atención especial en el pasado, ya que las materias primas para refractarios son de bajo costo, además del bajo costo de disposición final [1].

Debido a consideraciones medioambientales, el uso de material reciclado para sustituir materiales vírgenes puede reducir los desechos refractarios y puede tener un impacto positivo promoviendo el desarrollo sostenible con impacto económico, social y ambiental. Existe una amplia variedad de ladrillos refractarios (ASTM C27 - 98) específicamente formulados para cumplir con los requisitos particulares para diferentes industrias. Estos ladrillos se pueden clasificar según la composición química y el método de fabricación, entre otros criterios [4]. Una clasificación común de los refractarios es de acuerdo a su grado de porosidad [5]. Los materiales que contienen una alta fracción de poros se usan donde se requiere un buen aislamiento térmico a altas temperaturas, mientras que una baja porosidad se usa donde se requiere buena resistencia a la corrosión química. Del mismo modo, otras propiedades físicas, como la resistencia, la abrasión y la permeabilidad al gas, a menudo están relacionadas con la densidad y la porosidad del refractario.

Los refractarios a base de sílice y alúmina se utilizan ampliamente en la industria, debido a su extensa gama de aplicaciones y bajo costo, además de la posibilidad de usarse en ambientes ácidos [6]. El objetivo del presente estudio es evaluar la influencia de chamota, obtenida a partir de ladrillos porosos silico-alumisos que cumplieron su ciclo de vida en la industria cerámica, en las propiedades de un refractario como reemplazo de alúmina.

\section{Materiales y métodos}

Para evaluar la influencia de la chamota posconsumo en una pasta refractaria, se formularon cuatro mezclas como se muestra en la tabla 1. En estas se varió progresivamente el porcentaje en peso de chamota de $45 \%$ a $0 \%$ para hacer un análisis comparativo. La chamota posconsumo se obtuvo a partir de ladrillos porosos que ya habían cumplido su ciclo de vida en la industria cerámica. A estos se les realizó un proceso de conminución utilizando un molino de bolas cerámicas hasta llegar a obtener partículas $100 \%$ pasante malla 50 (abertura de malla: $297 \mu \mathrm{m}$ ). La chamota se caracterizó mediante espectroscopia de fluorescencia de rayos $X$ (Malvern Panalytical D-XRF ARL Optim'X).

Tabla 1. Formulaciones utilizadas para evaluar la influencia de la chamota posconsumo en una pasta refractaria como reemplazo de la alúmina. Los valores indican el porcentaje en peso.

\begin{tabular}{lcccl}
\hline \multicolumn{1}{c}{ Materias primas } & M1 & M2 & M3 & M4 \\
\hline Caolín & 30 & 30 & 30 & 30 \\
Arcilla & 15 & 15 & 15 & 15 \\
Alúmina & 10 & 25 & 40 & 55 \\
Chamota & 45 & 30 & 15 & 0 \\
\hline
\end{tabular}

La sinterización de las muestras se realizó en un horno a gas a una temperatura de $1600^{\circ} \mathrm{C}$. El cono pirométrico equivalente (CPE) se evaluó según la norma ASTM C-24. La densidad y la porosidad se determinaron siguiendo la norma ASTM C-20. La contracción lineal se determinó a partir de probetas con dimensiones de $50 \mathrm{~mm} \times 50 \mathrm{~mm}$ y un espesor de $10 \mathrm{~mm}$ antes y después del sinterizado. Las muestras se formaron utilizando la maquina universal de ensayos con ciclos incrementales de carga de $15 \mathrm{kN}$ hasta llegar a $62 \mathrm{kN}$ para formar la probeta. Previo a la compactación, la humedad de las muestras fue ajustada a $8 \%$. La dilatometría se realizó a las muestras sinterizadas en un dilatómetro NETZSCH Expedis Series DIL 402 desde $30{ }^{\circ} \mathrm{C}$ hasta una temperatura de $1500{ }^{\circ} \mathrm{C}$, a una velocidad de calentamiento de $5^{\circ} \mathrm{Cmin}^{-1}$.

\section{Resultados y discusión}

Los refractarios de alúmina pueden clasificarse de acuerdo al contenido de este material. Por ejemplo, los refractarios de arcilla típicamente tienen un rango de contenido de alúmina entre $25 \%$ y 45 $\%$ en peso; los refractarios de mullita tienen un contenido aproximado de alúmina de $63 \%$ y $37 \%$ de sílice en peso. Por otra parte, los refractarios de alúmina contienen un mínimo de $60 \%$ en peso de $\mathrm{Al}_{2} \mathrm{O}_{3}$, aunque el contenido puede ser $>99 \%$ para 
productos especializados. Un mayor contenido de alúmina proporciona una mejor resistencia a la fluencia y a la corrosión [4]. Los resultados del análisis por espectroscopia de fluorescencia de rayos $X$ de la chamota se muestran en la tabla 2 . La chamota utilizada corresponde a un ladrillo sílico aluminoso a base de arcilla, con 59,54\% de $\mathrm{SiO}_{2}$ y $30,25 \%$ de $\mathrm{Al}_{2} \mathrm{O}_{3}$.

Tabla 2. Composición química de la chamota obtenida por espectroscopia de fluorescencia de rayos X.

\begin{tabular}{cc}
\hline Óxidos & \% en peso \\
\hline $\mathrm{SiO}_{2}$ & 59,54 \\
$\mathrm{Al}_{2} \mathrm{O}_{3}$ & 30,25 \\
$\mathrm{CaO}$ & 2,76 \\
$\mathrm{~K}_{2} \mathrm{O}$ & 1,89 \\
$\mathrm{Fe}_{2} \mathrm{O}_{3}$ & 1,79 \\
$\mathrm{Na2O}$ & 0,618 \\
$\mathrm{LOI}$ & 0,51 \\
$\mathrm{MgO}$ & 0,47 \\
$\mathrm{SO}_{3}$ & 0,12 \\
Otros & 2,052 \\
\hline
\end{tabular}

El cono pirométrico equivalente, CPE, se utiliza ampliamente en la industria de los refractarios, ya que permite determinar el punto de ablandamiento de tales materiales [7]. Los resultados obtenidos para las distintas formulaciones indican que la muestra 1 tiene un cono pirométrico equivalente de 32; la muestra 2, uno de 34; y las muestras 3 y 4 , un cono superior a 36; esto revela que las temperaturas máximas de servicio son de $1717^{\circ} \mathrm{C}$, $1763{ }^{\circ} \mathrm{C}$ y mayores a $1804{ }^{\circ} \mathrm{C}$, respectivamente. Estas temperaturas son adecuadas para su aplicación como materiales refractarios. Hay que tener en cuenta que la chamota tiene un contenido de $\mathrm{K}_{2} \mathrm{O}$ del $1,89 \%$, el cual es un óxido alcalino $\mathrm{y}$ es un precursor de la formación de fases liquidas, ya que este reacciona con los silicatos de aluminio bajando el punto de fusión del material, efecto que se refleja en la reducción del CPE a medida que aumenta el contenido de chamota en la formulación del refractario [8].

Por otra parte, la dilatometría es una técnica que permite evaluar el comportamiento térmico de los cerámicos refractarios durante su uso. Las propiedades térmicas de los refractarios están dadas por el tipo y la cantidad de fases presentes a la temperatura de operación [9]. A partir del diagrama de fase de un sistema $\mathrm{Al}_{2} \mathrm{O}_{3}-\mathrm{SiO}_{2}[10]$, se observa que la mulita y el corindón son las fases más estables a temperaturas de servicio, y estas influencian las propiedades de los refractarios, además de la formación de fase vítrea.

En general, bajos contenidos de alúmina en la formulación del refractario llevan a la formación de mullita, mientras que altos contenidos de este óxido favorecen la formación de mulita y corindón, que, en este caso, aumenta la refractariedad del material obtenido [9]. En la figura 1 se pueden observar las diferentes curvas dilatométricas de las formulaciones; allí se tiene que el coeficiente de dilatación térmica cambia en función de la temperatura para cada una de las muestras. En general, en las figuras se puede observar una dilatación térmica entre la temperatura ambiente y $200{ }^{\circ} \mathrm{C}$ el cual puede estar asociado al cambio de cristobalita $\alpha$ a $\beta$ [11]. A partir de esta temperatura, los cambios pueden deberse a la reacción expansiva que se atribuye a la transformación de cuarzo $\alpha$ a $\beta$ la cual se lleva a cabo a partir de $573^{\circ} \mathrm{C}$ [12]; luego están el proceso de formación de fase vítrea alrededor de los $800^{\circ} \mathrm{C}$, el proceso de mullitizacion, y, en otros casos, a causa de la formación de corindón dependiendo de la formulación. Nótese que en las figuras 1a y 1b, las muestras que presentan mayor chamota tienen un cambio más pronunciado y este se debe al aporte que hace la chamota de sílice, la cual genera una mayor formación de mulita [13]. Por el contrario, en las figuras 1c y $1 \mathrm{~d}$, las curvas dilatometricas cambian debido a que se puede estar formando corindón, ya que estas formulaciones contienen una mayor proporción en peso de alúmina.

En la figura 2 se puede observar que el contenido de chamota afecta la contracción lineal de las muestras. A medida que se incrementa el porcentaje de chamota, las muestras tienen una mayor contracción. A partir de $15 \%$ de chamota, las muestras presentaron una expansión, debido a que el mayor porcentaje de chamota lleva a que, en la formulación final, haya más sílice, y este, al interactuar con la alúmina, forma mulita, la cual genera un cambio del volumen [14]. 


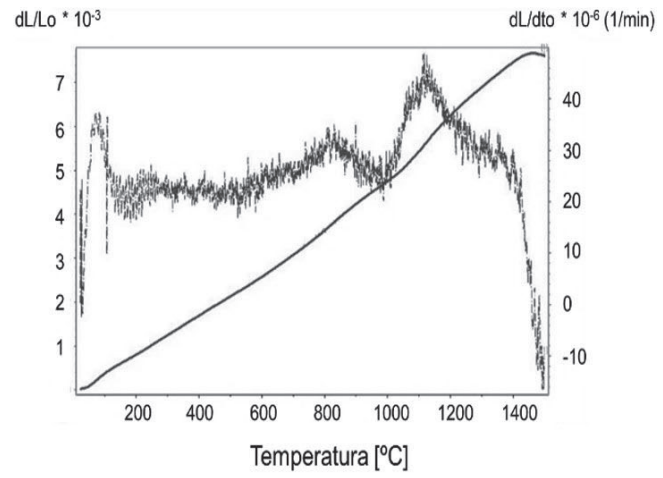

a.

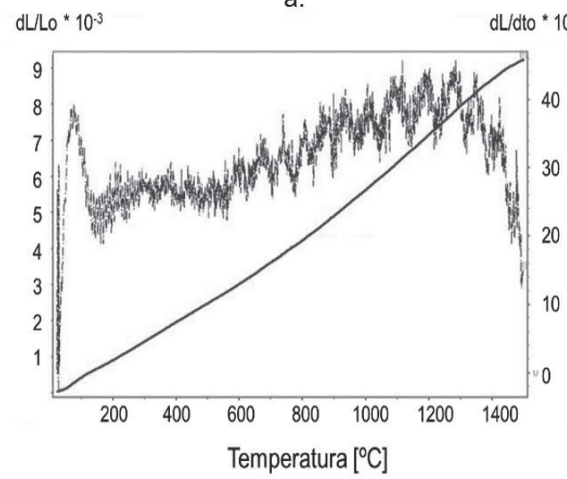

c.

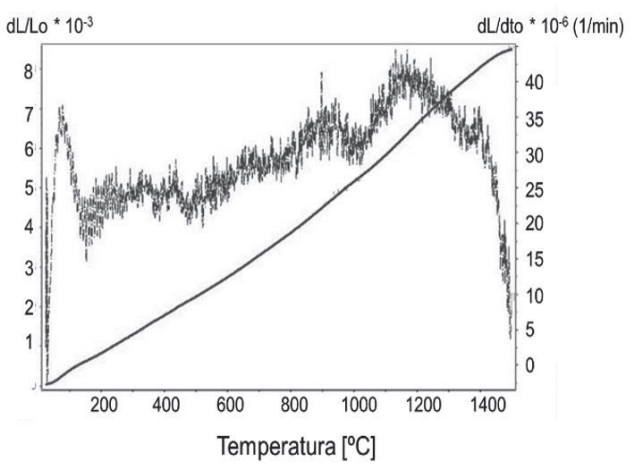

b.

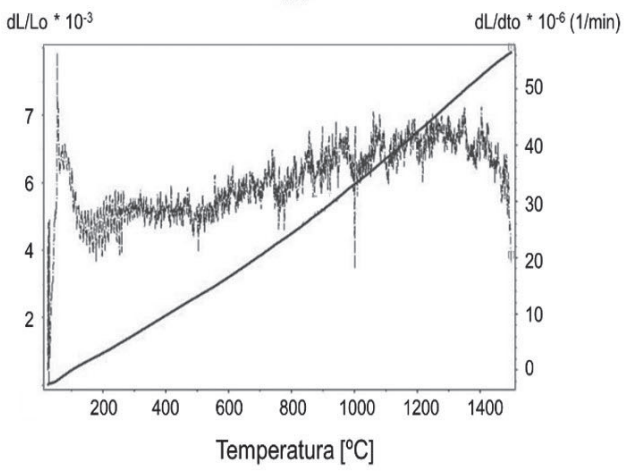

d.

Figura 1. Curvas dilatométricas: a. M1; b. M2; c. M3; d. M4.

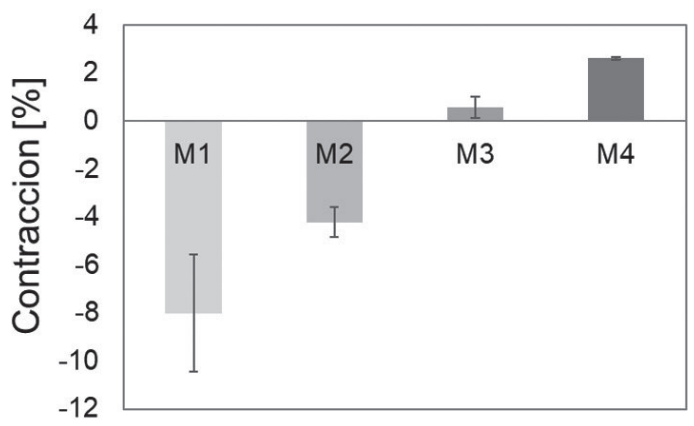

Figura 2. Contracción lineal de las muestras. Las barras de error indican desviación estándar.

Por otra parte, los efectos de la adición de chamota posconsumo sobre la porosidad y densidad se muestran en la figura 3. En la figura $3 a$ se puede observar cómo el porcentaje de chamota afecta la densidad de las muestras. Cuanto mayor sea la cantidad de chamota agregado a la pasta refractaria, mayor su densidad. En la figura 3b se observa que, a diferencia de la densidad, la porosidad disminuye cuando el porcentaje de chamota aumenta. Estos cambios en la densidad y porosidad son producto de la presencia de $\mathrm{K}_{2} \mathrm{O}$ al $1,89 \%$ el cual es un precursor de la formación de fases líquidas, lo que termina reduciendo la porosidad y aumentando la densidad; de esta manera también afecta ciertas propiedades, como la conductividad térmica efectiva (ke) que disminuye al incrementarse la porosidad, debido a que el aire actúa como una barrera frente al flujo de calor [15]. En general, con una apropiada proporción de chamota en la formulación de la pasta refractaria, se pueden lograr refractarios que tiene potencial aplicación cuando se requiere la formación de fases como la mulita, el corindón, o fases liquidas, además de aumentar la densidad del material refractario, brindando una mejor resistencia a la desintegración por choque térmico $[4,16]$. 


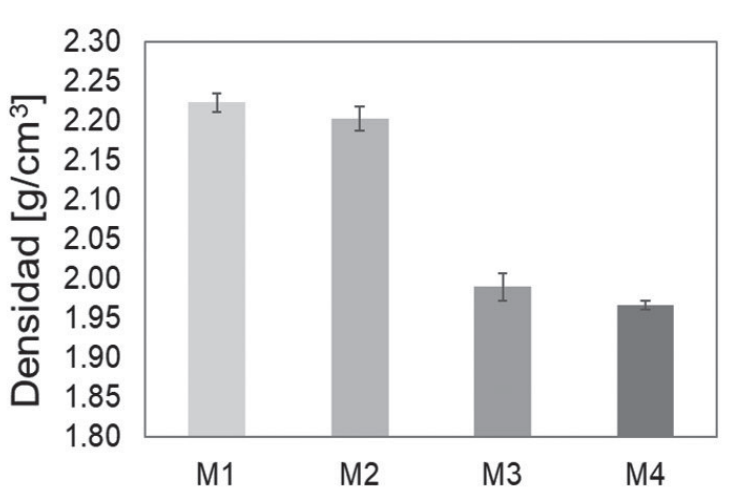

a.

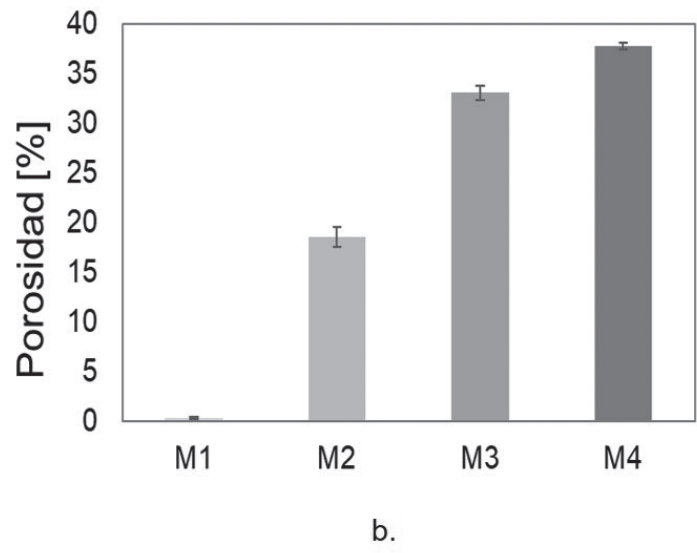

Figura 3. Densidad y porosidad a partir de norma ASTM C-20: a. Densidad; b. Porosidad. Las barras de error indican desviación estándar.

\section{Conclusiones}

En este estudio se logró evidenciar diferencias en las propiedades de los refractarios obtenidos a partir de las distintas formulaciones. Se encontró que, con la adición de chamota, aumentó la densidad de las muestras y disminuye la porosidad. Los resultados obtenidos para las distintas formulaciones indican que la muestra 1 tiene un cono pirométrico equivalente de 32 ; la muestra 2, de 34; y las muestras 3 y 4 un cono superior a 36; esto indica que las temperaturas de ablandamiento son de $1717{ }^{\circ} \mathrm{C}, 1763{ }^{\circ} \mathrm{C}$ y superiores a 1804 ${ }^{\circ} \mathrm{C}$, respectivamente, y son adecuadas para el uso como ladrillo refractario. Del mismo modo, las muestras con mayor contenido de chamota tienen un cambio más pronunciado en el ensayo de dilatometría debido al aporte que hace la sílice presente en la chamota que genera una mayor formación de mulita. Dado este comportamiento de las mezclas mediante la proporción de chamota en la pasta formulada, es posible modificar ciertas propiedades como la densidad, porosidad, temperatura máxima de trabajo, y conductividad térmica del refractario.

\section{Referencias bibliográficas}

[1] Horckmans L, Nielsen P, Dierckx P, Ducastel A. Recycling of refractory bricks used in basic steelmaking: A review. Resour., Conserv. Recycl. 2019;140:297-304.

[2] Nakamura Y, Hirai N, Tsutsui Y, Uchinokura $\mathrm{K}$, Tamura SI. Recycling of refractories in the steel industry. JAST. 1999; D693-D700.

[3] Segadães AM. Use of phase diagrams to guide ceramic production from wastes. Adv. Appl. Ceram. 2006;05(1):46-54.

[4] Sadik C, El Amrani IE, Albizane A. Recent advances in silica-alumina refractory: A review. J. Asian Ceram. Soc. 2014;2(2):83-96.

[5] Ishizaki K, Komarneni S, Nanko M. Porous Materials: Process technology and applications. United States: Springer US; 2013.

[6] Fang H, Smith JD, Peaslee KD. Study of spent refractory waste recycling from metal manufacturers in Missouri. Resour., Conserv. Recycl. 1999;25(2):111-124.

[7] Caniglia S, Barna GL. Handbook of industrial refractories technology: principles, types, properties and applications. United States: Noyes Publications; 1992.

[8] Carter CB, Norton MG. Ceramic materials: science and engineering. United States: Springer; 2007.

[9] Dana K, Sinhamahapatra S, Tripathi HS, Ghosh A. Refractories of alumina-silica system. Trans. Indian Ceram. Soc. 2014;73(1):1-13.

[10] Aramaki S, Roy R. Revised phase diagram for the system $\mathrm{Al}_{2} \mathrm{O}_{3}-\mathrm{SiO}_{2}$. J. Am. Ceram. Soc. 1962;45(5):229-242.

[11] Bauleke MP. How to solve the problems of body cracking and glaze popping in stoneware bodies. Bull. - Kans. Geol. Surv. 1977;(211):2327.

[12] Rossini L, Mennucci L, Figueras R. Dilatometría de arcillas refractarias argentinas. Bol. Soc. Esp. Ceram. 1970;9(5):507-538.

[13]Lee WE, Souza GP, McConville CJ, Tarvornpanich T, lqbal Y. Mullite formation in clays and clay-derived vitreous ceramics. J. Eur. Ceram. Soc. 2008;28(2):465-471.

[14] Fayyad SM, Al-Marahleh, GS, Abu-Ein, SQ. 
Improvement of the Refractoriness under Load of Fire-Clay Refractory Bricks. Adv. Theor. Appl. Mech. 2012;5(4):161-72.

[15]Rodríguez GP, Quintero JP, Tovar MG. Determinación Experimental de la Conductividad Térmica Efectiva en Bloques Extinguidos de Arcilla Roja. (Experimental
Determination of the Eective Thermal Conductivity in the Extruded Red Clay Bricks.). CeD [Internet]. 2014;5(1):15-20.

[16]Bertini J. Notas técnicas. Calidad de los refractarios. Revista IDIEM. 1965;4(3):189211. 